\title{
Development of Mucoadhesive Nanoparticulate System of Ebastine for Nasal Drug Delivery
}

\author{
Tashi Chhojom Khom, Hemant KS Yadav*, Abhay Raizaday, Navya Manne, \\ Hemant S Kumar and Sankeerth N Kumar \\ Department of Pharmaceutics, JSS College of Pharmacy, JSS University, Mysore, Karnataka -570015, India
}

*For correspondence: Email: haisunny2@yahoo.co.in; Tel: +91-9886112637; Fax: +9-0821-2548359

Received: 13 May 2013

Revised accepted: 26 April 2014

\begin{abstract}
Purpose: To prepare and evaluate mucoadhesive nanoparticulate system of ebastine for nasal drug delivery.

Methods: The nanoparticles were prepared by ionic gelation method using drug-chitosan weight ratios $1: 1,1: 2$ and $1: 3$, and incorporating 0.5 or $0.7 \%$ w/v sodium tripolyphosphate (STPP) and poloxamer 407. The mucoadhesive nanoparticles were characterized by scanning electron microscope (SEM), transmission electron microscope (TEM), differential scanning colorimetry (DSC) and Fourier transform infrared spectroscopy (FTIR) and evaluated for drug loading, entrapment efficiency, in vitro mucoadhesion, in vitro drug release and ex-vivo permeation.

Results: FTIR and DSC studies indicate that no chemical interaction occurred between the drug and polymer. Nanoparticle size ranged from 169 to $500 \mathrm{~nm}$. Drug loading and entrapment efficiency increased with increase in chitosan concentration and decreased with increase in poloxamer 407 concentration. The highest drug loading obtained for the nanoparticles was $19.5 \%$. With increase in polymer (chitosan) concentration (1:1 to 1:3), production yield was unchanged (73.2 to $74.4 \%$ (F6)). Mucoadhesion increased with increase in the concentration of chitosan. In vitro drug release from all the formulations was biphasic, being characterized by a slight 'burst' followed by slow release. At the end of 8 h F6 (1:3) showed drug release of only $86.9 \%$, indicating sustained release. Ex-vivo permeation of pure ebastine was more rapid than from F6, thus indicating the capability of chitosan to control drug permeation rate through sheep nasal mucosa.

Conclusion: The results indicate that a mucoadhesive nanoparticulate system can be used effectively for the nasal delivery of the antihistamine, ebastine.
\end{abstract}

Keywords: Chitosan, Ebastine, Mucoadhesive, Nanoparticles, lonotropic gelation, Permeation, Drug release, Poloxamer

Tropical Journal of Pharmaceutical Research is indexed by Science Citation Index (SciSearch), Scopus, International Pharmaceutical Abstract, Chemical Abstracts, Embase, Index Copernicus, EBSCO, African Index Medicus, JournalSeek, Journal Citation Reports/Science Edition, Directory of Open Access Journals (DOAJ), African Journal Online, Bioline International, Open-J-Gate and Pharmacy Abstracts

\section{INTRODUCTION}

Nasal administration can be used to deliver drugs for either local or systemic effect. The nasal route circumvents hepatic first pass elimination associated with oral delivery. Rapid mucociliary clearance of $t$ drug formulation is responsible for the low bioavailability of drugs administered by nasal route. To circumvent these problems, mucoadhesive drug delivery systems are being developed to provide a long term therapeutic concentration of the drug [1]. The use of mucoadhesive polymers for the development of delivery system maximizes the residence time of the drug formulation in the nasal cavity and hence prolonging the period of contact with the nasal mucosa thereby improving drug absorption [2]. 
Multiparticulate systems like nanoparticles, microparticles provide controlled release of the drugs. Nanoparticles are colloidal sized particles, possessing diameters ranging between 10 and $1000 \mathrm{~nm}$, and drugs may be encapsulated, adsorbed or dispersed in them. Properties of the nanoparticles are largely dependent on the polymers used to prepare it. Chitosan has been shown to have mucoadhesive properties because of its viscosity and interaction of the positively charged amino group with the negatively charged sites on the mucosa surface. Chitosan (CS) is a deacetylation derivative of chitin, and is biocompatible, biodegradable and non toxic in nature. Investigations have suggested that there are two effects of chitosan delivery systems on nasal mucosa. The mucoadhesive properties of the polymer can reduce the clearance rate of drugs from nasal cavity, thereby prolonging the contact time of chitosan delivery system with nasal epithelium. In addition, it has been shown that the interaction of the positively charged amino group of chitosan with the negatively charged sialic acid residues in mucus causes the transient opening of the tight junctions and allows large hydrophilic compounds to be transported across the epithelium [3]. Ebastine is a non sedating, second-generation $\mathrm{H} 1$ receptor antagonist. It is metabolized to its active metabolite, carebastine. It has antihistaminic and anti-allergic activities.

In the present research work, an attempt has been made to prepare mucoadhesive nanoparticulate system of ebastine for nasal delivery by ionic gelation method.

\section{EXPERIMENTAL}

\section{Materials}

Ebastine was obtained as a gift sample from Micro labs Ltd, Bangalore. Chitosan, Dialysis membrane (cut off mol. wt. 12000) and Poloxamer-407 was obtained from SigmaAldrich, Bangalore. Sodium tripolyphosphate STPP was obtained from Loba Chemie, Mumbai and Dichloromethane was obtained from Merck specialities Pvt Ltd, Mumbai. All the other solvents and chemicals used were of analytical grade.

\section{Preparation of nanoparticles}

The drug loaded nanoparticles were prepared by ionic gelation method [4]. The polymer; chitosan $(0.1,0.2$ and $0.3 \% \mathrm{w} / \mathrm{v})$ was dispersed in $100 \mathrm{ml}$ of $1 \%$ acetic acid solution under stirring for $4 \mathrm{~h}$ and stabilized overnight to obtain clear chitosan solution. The drug (Ebastine) was dispersed evenly in the chitosan solution using magnetic stirrer. Sodium tripolyphosphate solution (0.5 and $0.7 \% \mathrm{w} / \mathrm{v}$ ) and poloxamer 407 solutions (0.3 and $1 \% \mathrm{w} / \mathrm{v})$ were prepared in distilled water. Addition of $1.2 \mathrm{ml}$ of an aqueous sodium tripolyphosphate solution drop by drop to $3 \mathrm{ml}$ of chitosan solution at room temperature under high speed stirring $(15,000 \mathrm{rpm})$ using a homogenizer lead to the formation of chitosan nanoparticles. Finally, to stabilize the above nanoparticle suspension poloxamer-407 solution was added under high speed stirring. The resulting chitosan particle suspensions were centrifuged at 10,000 rpm for $15 \mathrm{~min}$ at $4{ }^{\circ} \mathrm{C}$. The nanoparticles thus obtained were washed with distilled water and freeze-dried.

\section{Characterization of nanoparticles}

\section{Fourier transform infrared (FT-IR) analysis}

The FT-IR spectra of pure drug (ebastine) and the formulation were recorded by $\mathrm{KBr}$ pellet method using FT-IR spectrophotometer (type $8400 S$ Shimadzu). Disappearance of ebastine peaks or shifting of peak in any of the spectra was noted.

\section{Differential scanning calorimetry (DSC)}

All dynamic DSC studies were carried out using DSC 60 Instrument. Colorimetric measurements were made with empty cell (high purity alpha aluminum discs) as the reference. The instrument was calibrated using high purity indium metal as standard. The dynamic scans were taken in nitrogen atmosphere at a heating rate of $10^{\circ} \mathrm{C} / \mathrm{min}$.

\section{Particle size, zeta potential and polydispersity index analysis}

The particle size, zeta potential and polydispersity index analysis of the nanoparticles were determined using Zetasizer (Malvern Worcestershire, UK). The nanoparticle dispersion was suitably diluted prior to particle size measurement at $25^{\circ} \mathrm{C}$.

\section{Scanning electron microscopy (SEM)}

The surface morphology of the formulation was determined using a scanning electron microscope (Joel SEM analysis instrument, Japan). Samples were mounted on aluminum mount using double-sided adhesive tape, sputtered with gold under vacuum and scanned at an accelerating voltage of $20 \mathrm{KV}$ before observation. 


\section{Transmission electron microscopy (TEM)}

The surface morphology of formulation was determined using a Transmission electron microscope. Samples were mounted on copper gridding, dried under vacuum and were scanned at an accelerating voltage of $15 \mathrm{KV}$ before observation.

\section{Determination of yield}

Determining whether the preparation procedure chosen for incorporating a drug into the polymers is efficient is of prime importance. The raw materials, amount of active compound, and other process parameters are deciding factors for the yield of the product during the preparation of nanoparticles. Yield was computed from Eq 1.

Yield $(\%)=\{(\mathrm{Wn} / \mathrm{Wp}+\mathrm{Wd}) 100$

where $W n$ is the weight of nanoparticle; $W p$ is weight of the polymer while $\mathrm{Wd}$ is the weight of drug.

\section{Assessment of drug loading and encapsulation efficiency}

To determine the amount of drug encapsulated in the nanoparticles, $100 \mathrm{mg}$ of the prepared nanoparticles was dissolved in $10 \mathrm{ml}$ of dichlromethane and diluted to $100 \mathrm{ml}$ using $\mathrm{pH}$ 6.4 phospate buffer. The above solution was centrifuged at $15,000 \mathrm{rpm}$ for $10 \mathrm{~min}$ in a cooling centrifuge and the amount of free drug in the supernatant was determined using UV-Visible Spectrophotometer (UV-1601, Shimadzu Co, Japan) at $257 \mathrm{~nm}$. The amount of drug encapsulated in the nanoparticles was calculated as the difference between the total amount of drug used to prepare the nanoparticles and the amount that was found in the supernatant, expressed as a percentage. Encapsulation efficiency (EE) was expressed as in Eq 2.

$\mathrm{EE}(\%)=(\mathrm{Dn} / \mathrm{Wn}) 100$

where $\mathrm{Dn}$ is the actual quantity of drug encapsulated and WN is the actual amount of drug used in preparing the nanoparticles

Drug loading (DL) was computed as in Eq 3.

$\mathrm{DL}(\%)=(\mathrm{Dn} / \mathrm{Nw}) 100$

where $\mathrm{Dn}$ is the actual quantity of drug found in the nanoparticles and Nw is the total weight of nanoparticles.

\section{In vitro mucoadhesion studies}

The mucoadhesion studies described in literature was used with slight modifications [5]. Sheep nasal mucosa was obtained from a local slaughter house, cleaned with distilled water and then cut into $2 \times 2 \mathrm{~cm}$ pieces for the study. The drug-loaded nanoparticles were immersed in 50 $\mathrm{ml}$ beaker at $37 \pm 0.5{ }^{\circ} \mathrm{C}$ containing $\mathrm{pH} 6.4$ phosphate buffer for 5 min in such a way that the solution just covered the nanoparticles. After the nanoparticles were wetted, the fresh sheep nasal mucosa was placed on the nanoparticles surface for $5 \mathrm{~min}$ so as to cover all the nanoparticles. The nasal mucosa with attached nanoparticles was removed and the remaining nanoparticles on the glass beaker were dried at $60{ }^{\circ} \mathrm{C}$. The percentage of adhered nanoparticles (AN) was computed as in Eq 4.

$\mathrm{AN}(\%)=\{(\mathrm{Wo}-\mathrm{Wr}) \operatorname{Wo}\} 100$

where $\mathrm{WO}=$ initial weight of the nanoparticles , $\mathrm{Wr}=$ unattached nanoparticles weight

\section{In vitro drug release studies}

The in vitro release studies were carried out using simple diffusion cell apparatus which is open at both the ends [6]. One end was tied with dialysis membrane (cut off mol.wt, 12,000) which serves as a donor compartment and the nanoparticles were placed on it. The dissolution medium used was $\mathrm{pH} 6.4$ phosphate buffer. Prior to the experiment the dialysis membranes were soaked overnight in the dissolution medium. This medium was stirred using a magnetic stirrer and the temperature was maintained at $37 \pm 0.5{ }^{\circ} \mathrm{C}$. Periodically, $1 \mathrm{ml}$ of sample was withdrawn and analysed spectrophotometrically at $257 \mathrm{~nm}$. Each time the sample was withdrawn, an equal amount of fresh dissolution medium was added to the receptor compartment to maintain sink conditions.

\section{Ex vivo permeation studies}

The permeation study was conducted using sheep nasal mucosa in a Franz type diffusion cell for $8 \mathrm{~h}$ [7]. Initially, $10 \mathrm{mg}$ drug-loaded nanoparticles (F6) or pure drug (ebastine) was placed on the mucosa in the donor compartment. The receptor compartment containing $\mathrm{pH} 6.4$ phosphate buffer maintained at $37 \pm 0.5^{\circ} \mathrm{C}$ was stirred constantly. At predetrmined time intervals, $5 \mathrm{ml}$ of sample was withdrawn from receptor compartment and analysed spectrophotometrically at $257 \mathrm{~nm}$. Each time the sample was withdrawn, an equal amount of fresh medium was added to the receptor compartment to maintain the sink conditions.

\section{Data analysis}

To determine the mechanism of drug release from the nanoparticles, the release data were 
fitted to zero-order, first-order, and Higuchi models using the PCP.Disso-V2.08 software. Comparison of the data obtained for F4, F5 and F6 was made by Student $t$-test at $95 \%$ level of confidence using Microsoft Excel 2007.

\section{Stability studies}

The optimized formulation were packed in a screw capped bottle and stability studies were carried out periodically over 90 days after storage at $25 \pm 2{ }^{\circ} \mathrm{C} / 60 \pm 5 \% \mathrm{RH}$ and $40 \pm 2{ }^{\circ} \mathrm{C}$ $175 \pm 5 \% \mathrm{RH}$.

\section{RESULTS}

\section{FT-IR analysis}

The characteristic IR absorption peaks of Ebastine at $1269 \mathrm{~cm}^{-1}$ (C-N stretch), $1450 \mathrm{~cm}^{-1}$ ( $C=C$ stretch), $1678 \mathrm{~cm}^{-1}$ ( $C=O$ stretch) and 3053 $\mathrm{cm}^{-1}$ (C-H stretch) were present in ebastine loaded chitosan nanoparticles. The FT-IR spectra of Ebastine pure drug and ebastine loaded chitosan nanoparticles are shown in Figure 1.

\section{Differential scanning calorimetry (DSC)}

From the DSC thermograms it was observed that, ebastine displayed a single sharp peak corresponding to its melting point at $87.74{ }^{\circ} \mathrm{C}$ and similar peak was observed at the same temperature in the formulation F6.

\section{Particle size, Zeta potential and PDI analysis}

The results shown in Table 1 indicated that increasing the chitosan concentration from 100 to $300 \mathrm{mg}$ at $0.7 \% \mathrm{w} / \mathrm{v}$ and $0.5 \% \mathrm{w} / \mathrm{v}$ STPP caused an increase in particle size from 310 to $500 \mathrm{~nm}$ and 169 to $280 \mathrm{~nm}$, respectively. It was observed that as the concentration of stabilizer decreased from 1 to $0.3 \% \mathrm{w} / \mathrm{v}$, particle size of the nanoparticles increased from 280 to $500 \mathrm{~nm}$. Zeta potential values of drug loaded NPs are shown in Table 1 ranging from $+18.9 \mathrm{mV}$ to + $32.6 \mathrm{mV}$ and the PDI values of the prepared formulations were found to be in the range of 0.7 to 1 .

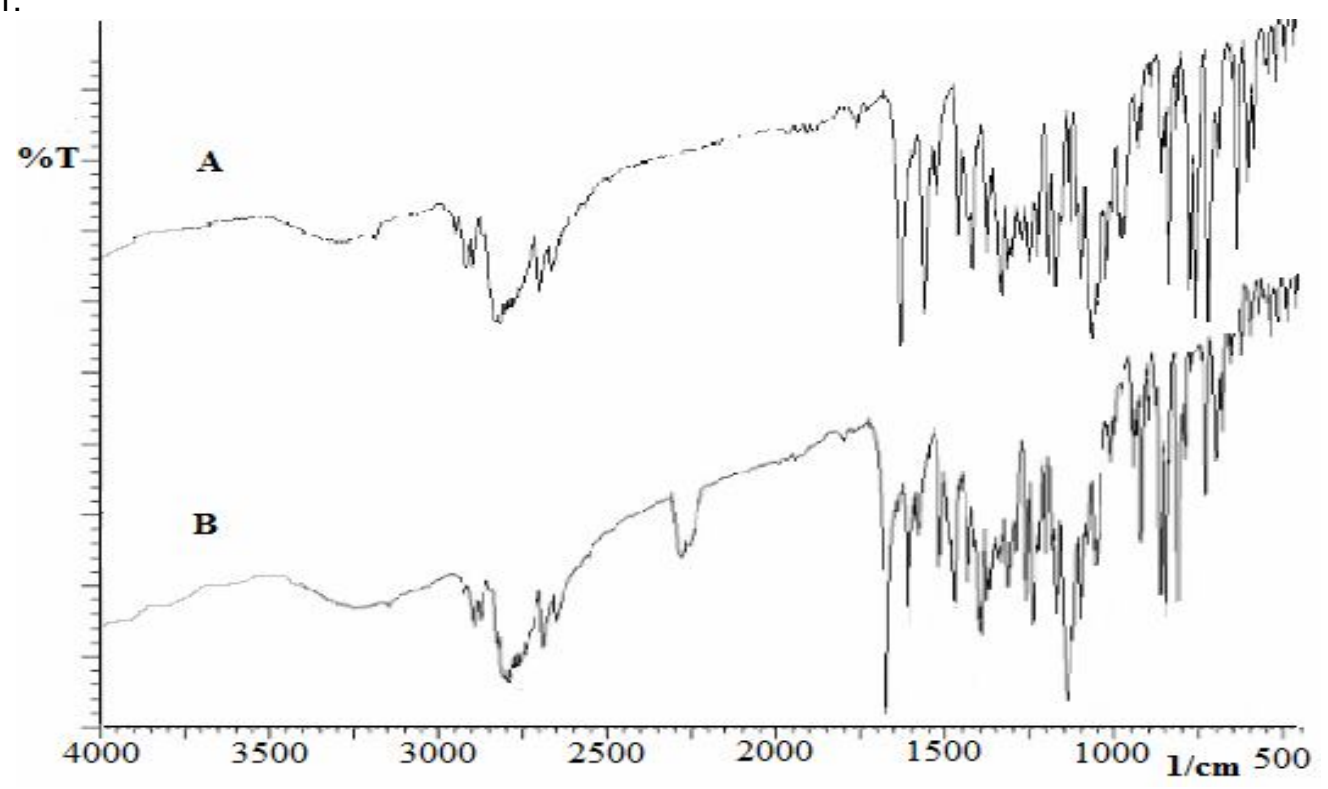

Figure 1: FTIR spectra of Ebastine (A) and Formulation F6 (B)

Table 1: Particle size, zeta potential and PDI of drug-loaded chitosan NPs

\begin{tabular}{lccc}
\hline Formulation code & $\begin{array}{c}\text { Particle size }(\mathbf{n m}) \\
\text { Mean } \pm \text { SD }\end{array}$ & $\begin{array}{c}\text { Zeta potential } \\
(\mathbf{m V})\end{array}$ & PDI \\
\hline F 1 & $310 \pm 2.8$ & +18.9 & 1.000 \\
F 2 & $392 \pm 5.1$ & +24.2 & 0.911 \\
F 3 & $500 \pm 4.0$ & +32.6 & 0.783 \\
F 4 & $169 \pm 3.2$ & +20.8 & 1.000 \\
F 5 & $234 \pm 4.8$ & +23.2 & 0.862 \\
F 6 & $280 \pm 1.6$ & +28.6 & 0.716 \\
\hline
\end{tabular}




\section{SEM and TEM}

SEM and TEM were carried out to study the morphology of nanoparticles. SEM and TEM images indicate that the nanoparticles have spherical shape, smooth surface and were in size range of $300 \mathrm{~nm}$. The SEM and TEM images are shown in Figure 2(a) and Figure 2(b) respectively.
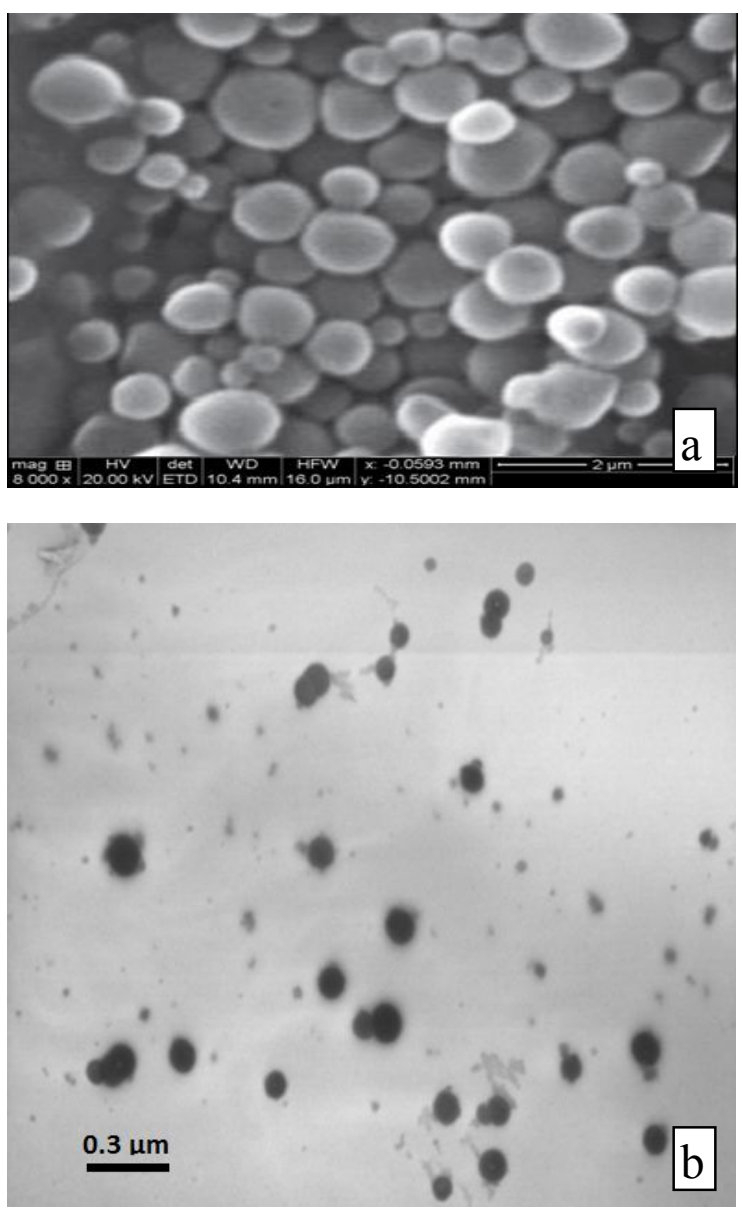

Figure 2: SEM (a) and TEM (b) images of drug-loaded chitosan NPs (F6)

\section{Production yield}

The production yield of NPs formulation F1 and F6 was 66.9 and $74.4 \%$, respectively. Increase in production yield was insignificant with the change in the polymer concentration $(p<0.05)$.

\section{Drug loading (DL) and encapsulation efficiency (EE)}

The data obtained from the results showed that as the polymer concentration increased there was an increase in the drug loading from 17.2 to $23.4 \%$ (F1 to F3) and 16.6 to $19.5 \%$ (F4 to F6). Drug loading also depended on stabilizer concentration. The data obtained from results shows that as the stabilizer concentration increased from 0.3 to $1 \% \mathrm{w} / \mathrm{v}$, drug incorporation was reduced from $23.4 \%$ to $19.5 \%$. The effect of stabilizer on drug loading was not significant $(p$ $<0.05$ ). The entrapment efficiency was found to increase with increase in polymer concentration. As the chitosan concentration increased from 100 to $300 \mathrm{mg}$, the entrapment efficiency increased from 38.4 to $44.4 \%$ (F1 to $F 3$ ) and 26.8 to $36.4 \%$ (F4 to $F 6$ ). The increase in stabilizer concentration from 0.3 to $1 \%$ lead to a decrease in the entrapment efficiency from 44.4 to $36.4 \%$.

\section{In vitro mucoadhesion study}

The results of in vitro mucoadhesion carried out showed that all the prepared formulation had good mucoadhesive property. It was found that increase in the concentration of chitosan in the formulation increased the mucoadhesion from 35.5 to $68.2 \%$ (F1 to F3) and 39 to $78.6 \%$ (F4 to F6). Formulation F6 showed maximum mucoadhesion $78.6 \%$.

\section{In vitro drug release study}

The in vitro release profiles of the drug loaded chitosan NPs were carried out in $\mathrm{pH} 6.4$ Phosphate buffer for $8 \mathrm{~h}$. Sustained release of the drug from the NPs is important as it would allow for a prolonged residence of the drug at the absorption site, increasing drug bioavailability. The data indicate that drug release from F6 was slower $(p<0.05)$ than from F4 and F5. The in vitro release data graph is represented in Figure 3.

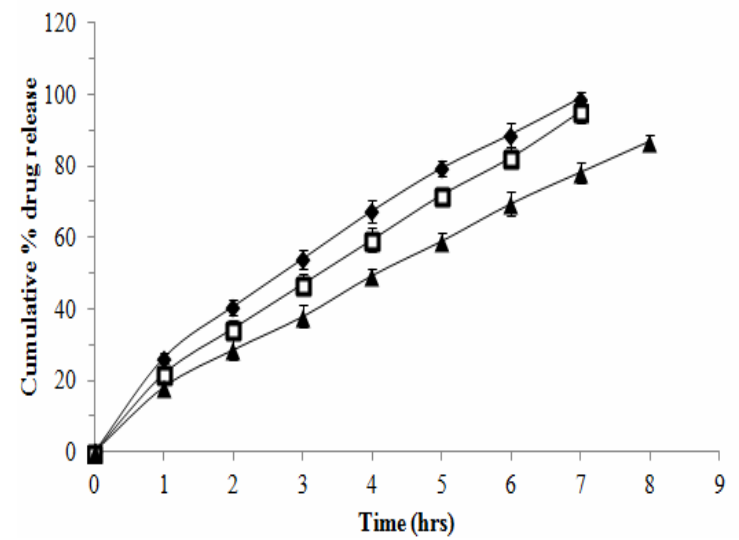

Figure 3: In vitro release studies of chitosan NPs

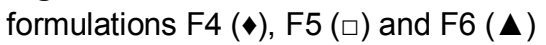

The release pattern of all the formulations was observed to be in a biphasic manner 
characterized by slight burst effect followed by a slow release. The in vitro release indicates that all the formulations (F4, F5 and F6) showed burst release within first $2 \mathrm{~h}$ followed by slow release for the next $6 \mathrm{~h}$. After $8 \mathrm{~h}$, formulations F4 and F5 released more than $95 \%$ of the drug while $\mathrm{F} 6$ showed a release of $86.9 \%$. This explains the sustained behavior of the formulation F6. Thus based on the drug release data F6 was selected as the optimized formulation for ex vivo study.

\section{Ex vivo permeation}

The ex vivo permeation study was carried out using sheep nasal mucosa. Formulation F6 was selected as the optimized formulation for further studies. The permeation profile of Ebastine from the formulation over an $8 \mathrm{~h}$ period is shown in Figure 4. The permeation of ebastine was rapid compared to optimized formulation (F6). The drug loaded chitosan nanoparticles showed sustained permeation profile.

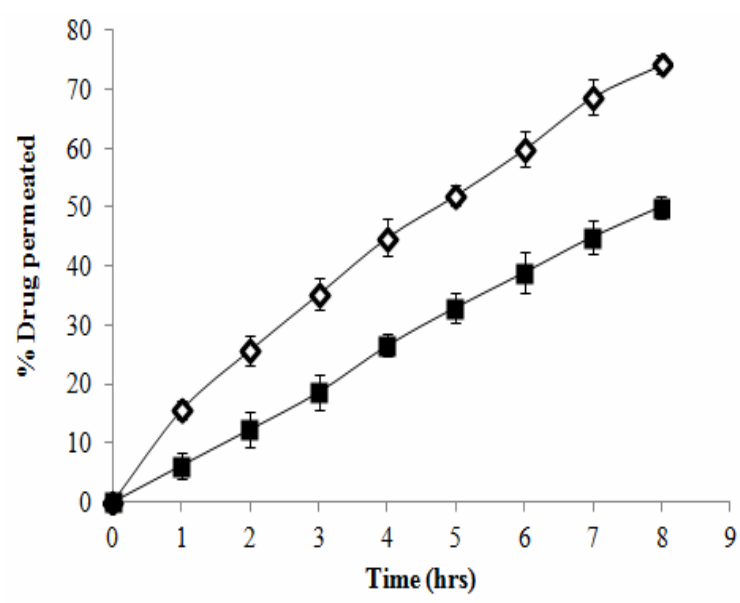

Figure 4: Ex-vivo permeation of pure ebastine $(\diamond)$ and F6 (घ) through sheep nasal mucosa

\section{Stability studies}

It was observed that there was no marked change in the physical property and the drug content of the optimized formulation during the stability study period.

\section{DISCUSSION}

The FT-IR spectra of ebastine pure drug and the optimized formulation (F6) indicate that characteristic peak of ebastine was not altered in their position after successful entrapment in the chitosan nanoparticles. Hence it can be inferred that there is no chemical interaction between the drug and polymer and it can be concluded that the characteristic bands of pure drug were not affected by the method used to prepare nanoparticles.

The DSC thermograms show that, ebastine displayed a single sharp peak corresponding to its melting point and similar peak was observed at the same temperature in the formulation F6. Hence it can be observed that there was no significant interaction between the drug and the polymers used.

The results shown in Table 1 indicate that by increasing the chitosan concentration at a constant STPP concentration, there was an increase in the particle size of the prepared nanoparticles. This can be explained by the fact that a lower concentration of gelation medium with lower concentration of chitosan results in a decrease in the liquid phase resistance against dispersion, forming smaller nanoparticles. According to Zeta potential reports in Table 1, chitosan nanoparticles are positively charged which can be explained by the particle formation mechanism, the positively charged amine groups are neutralized by their interaction with the negatively charged tripolyphosphate molecules. The residual amino groups would be responsible for the positive potential. This net positive charge of the nanoparticles is desirable to prevent particle aggregation and promote electrostatic interaction with the negative charge of the mucus layer. The higher zeta potential in a certain range implied that chitosan nanoparticles are stable in the formulation. It seems likely that the long amino groups hinder the anion adsorption and keep the high value of the electrical double layer thickness, suggesting the prevention of aggregation [8]. The mechanism of chitosan NP formation is based on electrostatic interaction between amine group of chitosan and negatively charged group of polyanion such as tripolyphosphate.

Polydispersity index is a parameter used to define the particle size distribution of NPs. The polydispersity index values should be between 0 and 1.0. If PDI value is less it indicates a narrow size range of particles and if PDI value is high, i.e., around 1.0, then the size distribution of particles falls within a wide range. Homogeneous dispersions have a PDI value close to zero while PDI values greater than 0.3 suggest heterogeneous dispersion. The PDI value of formulation $\mathrm{F} 6$ was 0.716 .

SEM and TEM images indicate that the nanoparticles have a spherical shape, smooth surface and were in size range of $300 \mathrm{~nm}$. The 
drug loading and encapsulation efficiency was found to increase with increase in the polymer concentration. The increase drug loading and entrapment efficiency may be due to the greater proportion of polymer with respect to the amount of drug. The drug loading was also affected by the stabilizer concentration. With the increase in stabilizer concentration, drug loading and entrapment efficiency decreased as a result of interaction between drug and stabilizer.

The formulation had good mucoadhesive property. Increase in the concentration of chitosan in the formulation increased mucoadhesion. This may be due to the formation of secondary chemical bonds such as hydrogen bond or ionic bond or ionic interactions between the positively charged amino groups of chitosan and the negatively charged sialic acid residue of mucus glycoproteins or mucins. Sialic acid carries a net negative charge and providing strong electrostatic interaction between mucin and chitosan. Formulation F6 showed maximum mucoadhesion of $78.6 \%$.

The release pattern of all the formulations was observed to be biphasic characterized by slight burst effect followed by a slow release. The burst effect corresponds to the release of the drug located on or near surface of nanoparticles or release of poorly entrapped drug. The slow release may be due to the slow diffusion of the medium into the polymer matrix whereby degradation of the polymer occurs and drug diffuses out of the nanoparticles. Formulations $\mathrm{F} 4, \mathrm{~F} 5$ and $\mathrm{F} 6$ showed burst release within the first $2 \mathrm{~h}$ followed by slow release for the next $6 \mathrm{~h}$. The burst release of the formulations was due to the drug located on or near the surface of the nanoparticles.

When the in vitro release data were fitted into various kinetic models to determine the best-fit model, the results obtained indicate that the bestfit model was zero order. In all the cases, the value of ' $n$ ' was between 0.5 and 1 . This indicates that the release of drug from all the formulations was by anomalous transport. The drug loaded chitosan nanoparticles showed sustained permeation profile by the diffusion of the media.

\section{CONCLUSION}

Chitosan NPs exhibit significant mucoadhesive properties and could potentially be used for sustained intranasal delivery of antihistaminic drugs.

\section{ACKNOWLEDGEMENT}

The authors thank JSS University, Mysore and J.S.S. Mahavidyapeetha, Mysore, India for their invaluable support.

\section{REFERENCES}

1. Sanjay D, Beduin M, Bhasakar M, Ananya M, Sandeepan $D$. Nasal drug delivery: An approach of drug delivery through nasal route. Der Pharmacia Sinica 2011; 2(3): 94-106.

2. Shivam U, Ankit $P$, Pratik J, Upadhyay U, Chotai $N$. Intranasal drug delivery system- $A$ glimpse to become maestro. J Appl Pharm Sci 2011; 1 (3): 34-44.

3. Schipper N, Olsson S, Hoogstraate J, DeBoer A, Varum $K$, Artursson P. Chitosan as absorption enhancers for poorly absorbable drugs: Mechanism of absorption enhancement. Pharm Res 1997; 14: 923-929.

4. Quintanar-Guerrero D, Alle mann E, Fessi H, Doelker E. Preparation techniques and mechanism of formation of biodegradable nanoparticles from preformed polymers. Drug Dev Ind Pharm 1998; 24: 1113-1128.

5. lliger SR, Demappa T. Formulation and characterization of mucoadhesive microspheres of promethazine hydrochloride for nasal delivery. J Pharm Res 2011; 4(1): 276-279.

6. Umasankar K, Uma M. Formulation and Evaluation of Cytarabine Nanoparticles. Int J Innov Pharm Res 2010; 2: 48-52.

7. Barbara L, Federica B, Giuseppe C. Albumin nanoparticles carrying cyclodextrins for nasal delivery of the anti-Alzheimer drug tacrine. Eur J Pharm Sci 2011; 44: 559-565.

8. Xing $T$, Xiaomei $W, \mathrm{Na}$ C. Preparation of estradiol chitosan nanoparticles for improving nasal absorption and brain targeting. Eur J Pharm Biopharm 2008; 70: 735-740.

9. Paulo C, Jose Manuel SL. Modeling and compaction of dissolution profiles. Eur J Pharm Sci 2001; 13: 123133. 